\title{
A MYXOMATOUS TUMOUR OF PLEURO-PULMONARY ORIGIN
}

\author{
BY \\ H. BARKLEY AND R. H. CARDOZO \\ From St. Mary's Hospital, London, W.2
}

(RECEIVED FOR PUBLICATION APRIL 2, 1957)

Although myxomatous change in mesodermal tumours is a common finding, tumours consisting entirely of myxomatous tissue are much rarer. Stout (1948) reviewed the literature on myxomata and the cases seen at Columbia University, and found a total of 140 cases excluding those in the heart. None of these was in the lung or pleura. Mesodermal tumours in these sites are not uncommon and a number of these have shown myxomatous changes, but the only purely myxomatous tumour reported appears to be the case of Miller and Jackson (1954). This was a myxosarcoma which had caused arterial embolism. The authors suggested that this might possibly have arisen as a result of extensive myxomatous change in a chondromatous hamartoma.

The purpose of this paper is to describe the clinical and pathological features in a further case of a myxomatous tumour in the lung and pleura.

\section{CASE History}

H. R., a 54-year-old married professional woman, enjoyed excellent health until six months before admission, when, while driving her car, she developed stiffness of the left side of her neck with pain beginning under her left breast, radiating to her left shoulder, and spreading down the left arm. The pain was continuous, deep, and piercing, and was relieved by aspirin and codeine. It was aggravated by breathing but not by coughing. She had a history of minor cough for years with grey sputum, but this condition had not increased. Her past history was not contributory except that she had had a panhysterectomy for fibromyomata and an ovarian cyst in 1951 . She had smoked approximately 15 cigarettes a day for many years.

She was seen in the Out-patient Department where the only abnormal findings were some tenderness over the chest wall posteriorly at the level of $T$ 8-9 on the left side together with pain on movement of the spine. Radiological examination of the chest at that time showed an area of collapse with consolidation and effusion at the left base (Fig. 1). She was given penicillin for 10 days during which time the pain disappeared, but the radiograph remained unchanged. The white blood cell count was $9,000 / \mathrm{mm}^{3}$ ( $50 \%$ polymorphs, $49 \%$ lymphocytes, and $1 \%$ monocytes). Aureomycin was given, 0.25 g. q.i.d. for five days, when a radiograph showed that the consolidation and effusion had cleared revealing a rounded opacity anteriorly, adjacent to the left border of the heart (Figs. $2 A$ and $2 B$ ). She was therefore admitted for further investigation. Physical examination upon this admission revealed some irregularity of the spines of $\mathrm{T} 8-10$, but no tenderness. A few medium rales were heard at the left base. There was no widening of the area of percussion dullness to the left of the sternum. There were no other positive findings.

Laboratory investigations at that time revealed a haemoglobin of $14.8 \mathrm{~g} . \%$ and a white cell count of $8,000 / \mathrm{mm}^{3} .^{3}(37 \%$ polymorphs, $60 \%$ lymphocytes, and $3 \%$ monocytes). The urine was unremarkable. The E.S.R. was $78 \mathrm{~mm}$. $/ \mathrm{hr}$. (Westergren). A smear of her sputum showed no acid-fast bacilli, but culture produced a mixed growth containing moderate numbers of pneumococci. The serum alkaline phosphatase level was 5 King-Armstrong units.

The patient was reluctant to undergo bronchoscopy, and was discharged to the Out-patient Department. She was not seen again for four months, when she was referred by her family doctor because the size of the radiological opacity had increased. She had felt entirely well in the meantime. On examination at her second admission, she was a tanned, fit-looking woman appearing younger than her stated age. Her temperature was $98.4^{\circ} \mathrm{F}$., pulse 68 , respiration 18 , blood pressure $110 / 75 \mathrm{~mm}$. Hg. Physical findings were entirely normal. Laboratory investigations included a haemoglobin of $17.0 \mathrm{~g} . \%$. A chest radiograph revealed a circumscribed opacity at the left base anteriorly slightly larger than on the previous study suggestive of an encysted effusion, though the possibility of new growth could not be ruled out. Thoracotomy was performed on October 25,1955 , as a left postero-lateral approach through the fifth intercostal space. There were no adhesions except at the tip of the lingula which contained the main tumour. The surface of both lobes and the diaphragm was studded with nodules of amber encapsulated material attached to the pleural surface by tiny pedicles. Each was flattened to conform to the opposing pleural surface, was apparently covered by serosa, and had its blood supply clearly visible in its filamentous pedicle. Their frequency increased towards the base, but some could be found over the apex. The pedicles were more prominent on those attached to the visceral than to the parietal pleura where they tended 


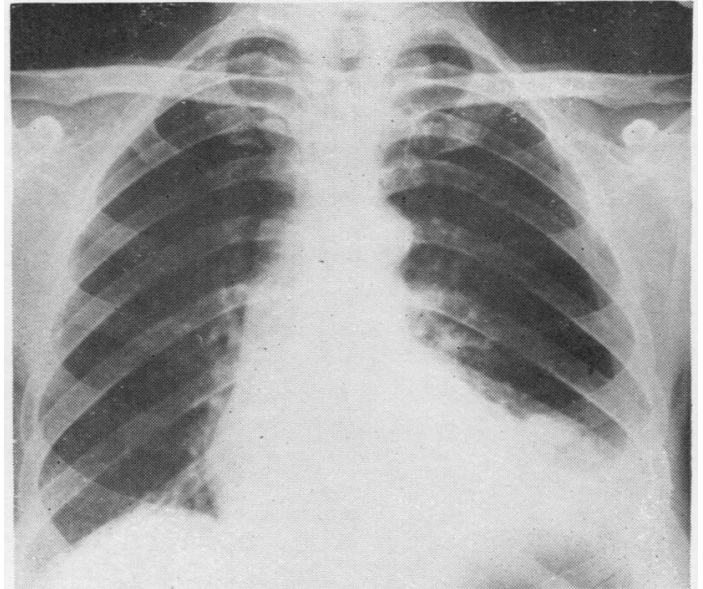

FIG. 1.-Radiograph on first admission. Area of collapse with consolidation and effusion at the left base.

to be sessile, especially over the dome of the diaphragm. These varied in size from barely visible to $1.5 \mathrm{~cm}$.

The main lesion at the tip of the lingula had the same appearance. It was globular, about $3 \mathrm{~cm}$. in diameter, and appeared like encapsulated fluid. It was translucent amber in colour. It arose in the extremity of the lingula, appeared to have invaded the anterior and

Figs. 2A and 2B.-Two weeks later, after treatment with antibiotics. Rounded opacity adjacent to the left border of the heart.

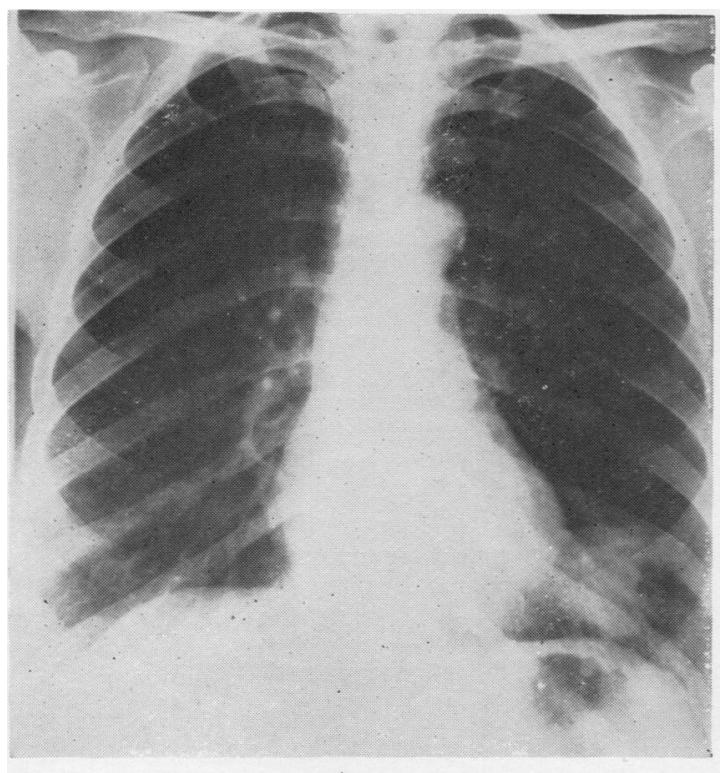

phrenic pleura and had grown into the pericardium as far back as the pericardiophrenic bundle from which it had to be excised by sharp dissection.

There was no evidence of hilar or mediastinal lymph node involvement and no other abnormal pulmonary parenchyma. Because of the multiple pleural nodules and the absence of hilar involvement, no attempt at radical pulmonary extirpation was made. After dissecting the lesion away from the chest wall, diaphragm, and mediastinum, a simple wedge resection of the terminal lingula was carried out and the stump oversewn. A lateral drainage tube was placed through the chest wall and the chest closed. Post-operatively her course was uneventful, and she was discharged on the eleventh post-operative day. She has been followed up in the Out-patient Department for 15 months and at the present time is asymptomatic. The radiograph (Fig. 3) shows an elevated fixed left diaphragm with some pleural thickening at the left base.

\section{Pathology}

Gross Appearances (Fig. 4).-The excised specimen consisted of a portion of lingula $7 \times 5 \times 3 \mathrm{~cm}$., containing a well-encapsulated, somewhat lobulated mass, $3 \mathrm{~cm}$. diameter, consisting of uniformly pale amber, soft, rather slimy material. No cystic areas or areas of haemorrhage or necrosis were seen. The adjacent lung was collapsed and showed no evidence of invasion.

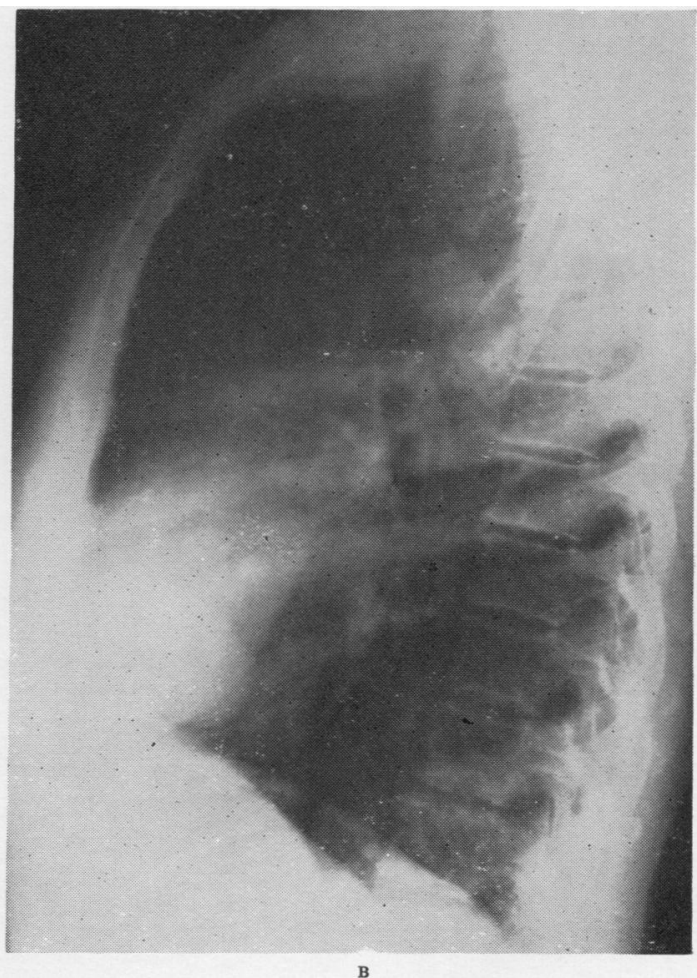




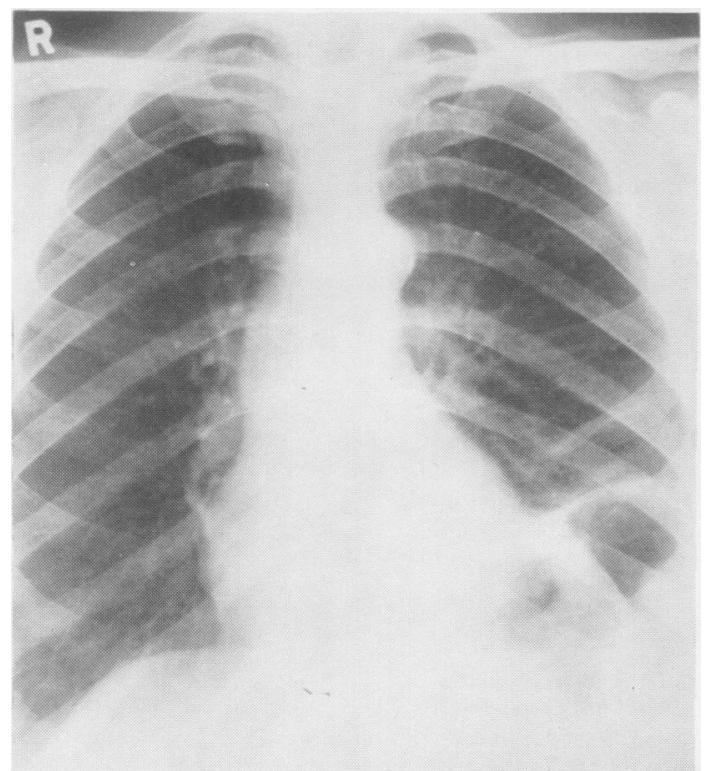

FIG. 3.-Fifteen months after operation, the left diaphragm is elevated and fixed. Some pleural thickening present at left base.

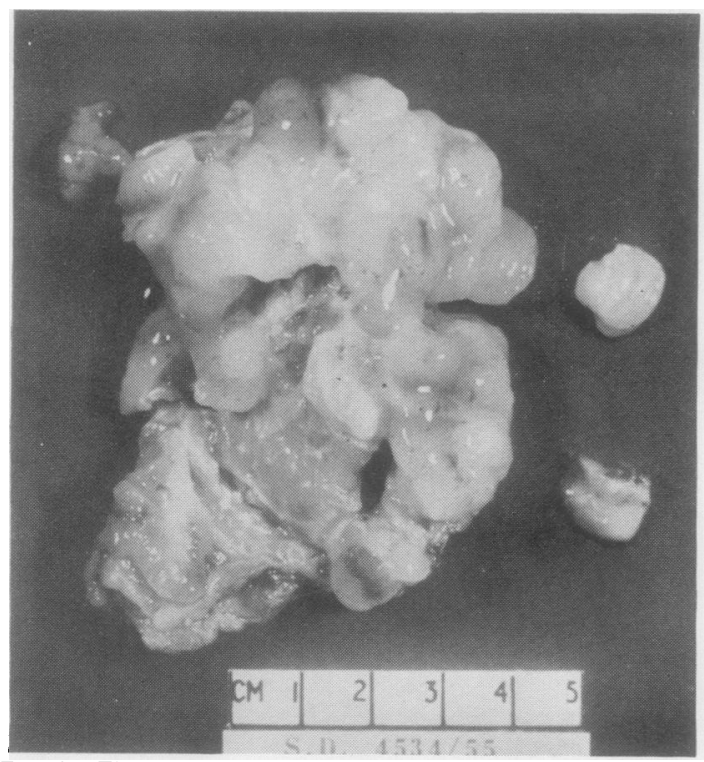

Fig. 4.-The main tumour (cut open) and two pleural seedlings.

Several small nodules from the pleura, identical in appearance with the main tumour, were also received.

Microscopic Appearances (Fig. 5).--Sections showed a uniform picture of cells arranged in small groups or strands, or singly, in an abundant vacuolated homogeneous stroma which stained bluish or pinkish with haematoxylin and eosin. There was some cellular

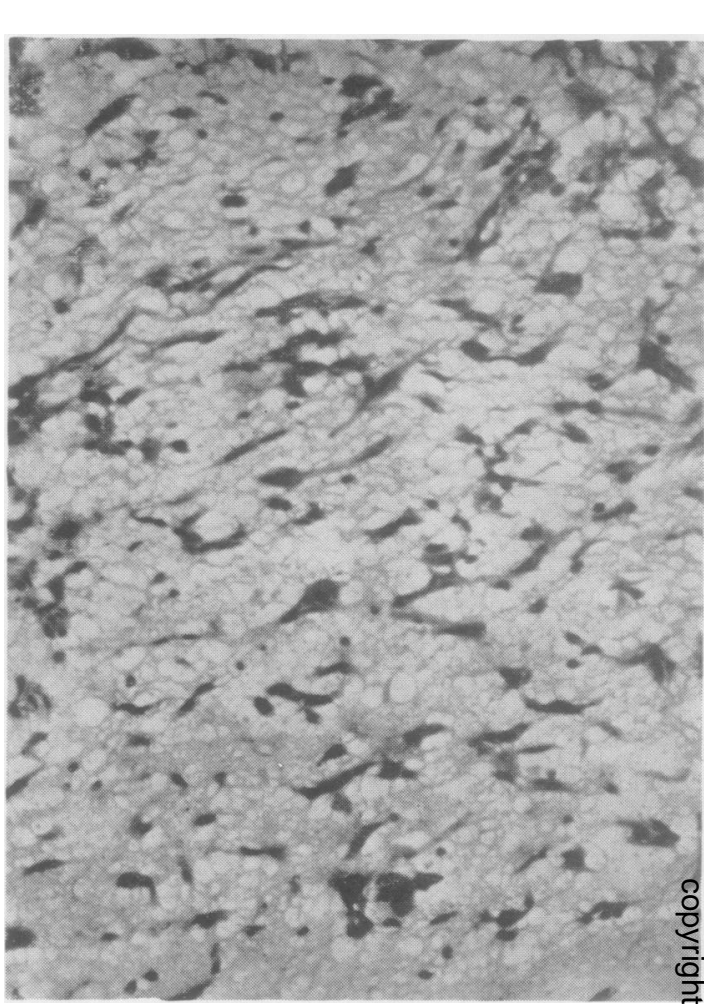

Fig. 5.-Photomicrograph of tumour.

pleomorphism, but most of the cells were stellate with an elongated or irregularly rounded outline. The most characteristic cell was composed of a well-defined, moderately hyperchromatic elliptical nucleus with a stellate homogeneous acidophil cytoplasm, which was not very clearly defined, and often appeared to merge with the surrounding stroma. Some of the cells appeared joined together by their cytoplasmic processes. Not infrequently the nuclei, and sometimes the cytoplasm, showed vacuolation. Mitoses were extremely rare. The stroma gave positive results with the usual stains for mucin, but there was no evidence of fat (Sudan III and osmic acid) nor of muscle cells (lissamine). Haematoxylin and van Gieson stain showed the presence of fairly scanty fine collagen fibres in the stroma, and silver impregnation revealed abundant argyrophil fibres both fine and coarse, but with little in the way of apparent anastomosis. Around the neoplastic deposits was a thin, rather loose fibrous capsule. In a few places, this appeared to be absent, but even so there was no evidence of cellular spread outside the main tumour masses, which everywhere had a clearly demarcated edge. Considerable pleural thickening was present in the vicinity of the growth. In some sections ill-defined fibrous trabeculae were present, often dense and somewhat hyaline, generally with moderate-sized blood vessels running through them. There were, however, no fibromatous areas. 
Blood vessels, both thin-and thick-walled, were present, but these were only moderately prominent. No evidence of invasion of blood vessels was seen.

Scattered throughout the stroma were moderate numbers of round cells and polymorphs.

\section{Discussion}

The presence of myxomatous tissue in a tumour is generally thought to be due to a degenerative change, and most myxomata are believed to result from this change in such tumours as fibromata, lipomata, chondromata, or their malignant counterparts. Many, in fact, believe that myxomata do not exist as a separate entity. It is therefore essential, when examining a myxomatous tumour, to search for evidence of a parent tumour. Examination of numerous sections has, in this case, failed to reveal any such evidence. No fibromatous or cartilaginous areas or fat have been seen, nor do any of the cells appear to be of muscular origin. The lesion would therefore seem to be either a true myxoma or to have arisen from a parent tumour, all evidence of which has been obliterated by myxomatous change. If the latter interpretation is the correct one, the possibility that the growth is metastatic in origin cannot be excluded, although clinically, up to the present time, there has been no evidence of a primary tumour elsewhere in the body.

The question arises as to whether this tumour is benign or malignant. Stout (1948) is doubtful whether there is any value in dividing the tumours into myxomata and myxosarcomata on the grounds that. although they may invade locally, they do not metastasize, and he considers that one cannot anticipate the growth energy of the tumour from histological examination. Others, however (Sponsel, McDonald, and Ghormley, 1952), believe that a histological distinction can be made and that this is helpful in prognosis.
In this case the histological features suggest a benign rather than a malignant tumour, but the numerous pleural nodules require an explanation. These might be pleural seedlings from the main tumour, and their position in the lower part of the pleural cavity, with diminution in frequency, passing towards the apex, suggests that gravity may have played some part in determining their site. They may, on the other hand, be explained in terms of a multifocal origin. Certainly from a clinical point of view the growth, at the present time, appears to be pursuing a benign course, for, although much tumour was left untouched at operation, when the patient was last seen she was symptomless and the chest radiograph showed no new development.

\section{SUMMARY}

A case is described of a middle-aged woman who presented with sudden pain in the chest. Radiographs showed collapse, consolidation, and effusion at the left base. These findings cleared with antibiotic therapy, and radiographs revealed a rounded opacity close to the left border of the heart. At thoracotomy a myxomatous tumour in the lingula and numerous similar pleural nodules were found. The main tumour and several pleural nodules only were excised. The pathological findings are described and discussed.

At the present time, 15 months after operation, the patient is quite symptomless, and there has been no change in the radiological appearances since operation.

We would like to thank Mr. L. L. Bromley for permission to publish this case.

\section{REFERENCES}

Miller, A. A., and Jackson, F. B. (1954). J. Path. Bact., 68, 221. Sponsel, K. H., McDonald, J. R., and Ghormley, R. K. (1952). J. Bone Jt Surg., 34A, 820.

Stout, A. P. (1948). Ann. Surg., 127, 706. 